\title{
Details of temperature compensation for strain measurements on NPL bridge - demonstrator for SHM
}

\author{
E. N. Barton ${ }^{1, a}$, B. Zhang ${ }^{1, b}$ \\ ${ }^{1}$ National Physical Laboratory, \\ Hampton Rd, Teddington, Middlesex, UK TW11 OLW \\ aelena.barton@npl.co.uk, bbufa.zhang@npl.co.uk,
}

Keywords: bridge monitoring, temperature compensation, fibre Bragg gratings, solar radiation sensors, structural health monitoring

\begin{abstract}
This paper is part of ongoing structural health monitoring in the National Physical Laboratory. The work is aimed at improving reliability of sensors and increasing the uptake of monitoring systems by improving the understanding of the fundamental interactions between the sensors and their environments. It is important to optimize and validate the selection of the sensors for each application in order to ensure that the results are meaningful. An example of unexpected aspects of a sensor performance exposed to temperature and solar radiation is demonstrated and the importance of temperature compensation is highlighted. More work is required fully to explain this phenomenon.
\end{abstract}

\section{Introduction}

A 15 tonne, 5 metre high, 20 metre long reinforced concrete footbridge is one of the largest specimen ever created at NPLand is ideal as a demonstrator for this project. Built in the mid 1960s and used for more than 40 years, the bridge has been moved and more than 12 different techniques have been selected for long-term structural health monitoring. In order to capture as much data as possible during the controlled damage and repair tests on the bridge, and to showcase several different available technologies, the footbridge has recently been covered with a variety of sensors, both traditional and novel, and measured externally using a wide range of techniques. Examples of techniques are listed below in alphabetical order:

3D Laser Scan provided during the tests by Dr N Eden from John Moore University,

Acoustic Emission to detect the formation of cracks, supplied and installed by Physical Acoustics and Dr R Pullin from Cardiff University,

- $\quad$ Digital Image Correlation widely used in NPL by Dr N McCormick,

- Digital Leveling of foundations performed before and after each test by Smithers Purslow Property Services,

Electrolevel Beam Sensors measuring tilt, provided and installed by Soil Instruments,

Fibre Bragg Gratings written by City University and patches installed by Smart Fibres

Resistance Strain Gauges in the form of standard Vishay gauges, installed by NPL experts

Time-Domain Reflectometry Distributed Crack Sensor provided by Strainstall

Vibrating Wire Strain Gauges measuring strain on both sides of the deck, installed by Soil Instruments,

Video Gauge Technique to measure displacements during the tests using Imetrum cameras

Wireless Accelerometer Sensors used as 2 dimensional tilt sensors supplied by Sencieve Services

Wireless Magnetic Induction Sensors using SIM cards from Smithers Purslow Property

- Numerous environmental sensors including a weather station and surface wetness sensors from Delta-T Devices, and solar radiation sensors from Campbell Scientific. 


\section{Strain Measurements.}

Various strain sensors have become widely available for structural health monitoring, especially in civil infrastructures. Most of these devices cannot sense strain and temperature independently. Therefore, their temperature response and mechanisms for its compensation are of critical concern for externally installed sensors.

\section{Temperature Variation due to Sensor Location.}

During our monitoring program, significant daily temperature variations of about $15-20{ }^{\circ} \mathrm{C}$ on average were observed between day and night and about $5{ }^{\circ} \mathrm{C}$ between the sunny and shady sides due to location of the bridge on NPL site, close to the parkland.

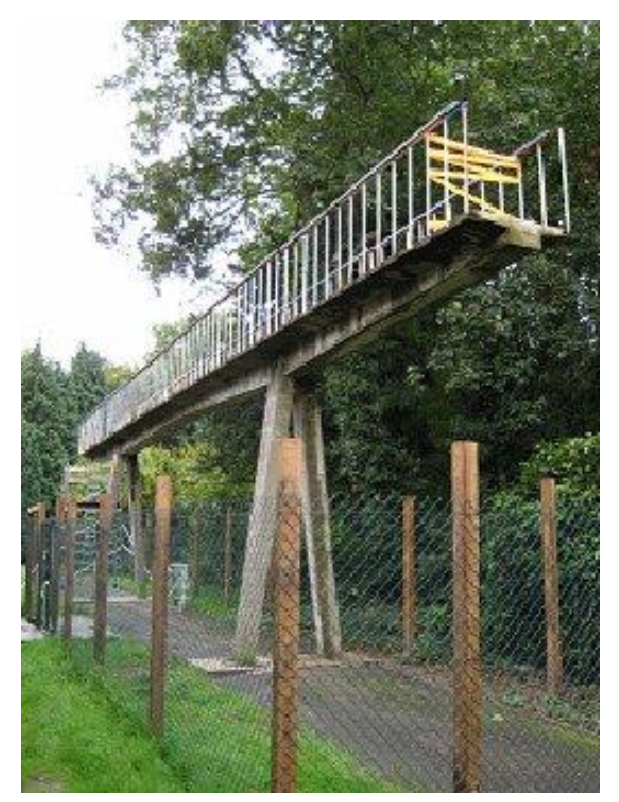

Fig. 1. Bridge location showing an open front side and parkland.

Having relatively slender dimensions, with the deck being about $950 \mathrm{~mm}$ wide and $350 \mathrm{~mm}$ thick, the thermal load was very significant, as shown experimentally and supported by modeling [1], [2]. For example, a vertical displacement at the end of the cantilever due to average daily temperature variation of $2.7 \mathrm{~mm}$ was approximately equivalent to an applied vertical load of $4.5 \mathrm{kN}$ at the free end of the cantilever [3].

Among hundreds of other sensors the FBG patches were installed on the bridge in the locations shown on Fig. 2. Each patch contained two gratings: one to measure strain and the other to provide temperature measurement for temperature compensation. It is clear from Fig. 3 that the sensors 3, 4, 5,6 and 7, on the top of the deck, are fully exposed to the sun and the sensors 1,2, 8, 9 and 10, in shady areas, are protected from the direct sunlight most of the time. 


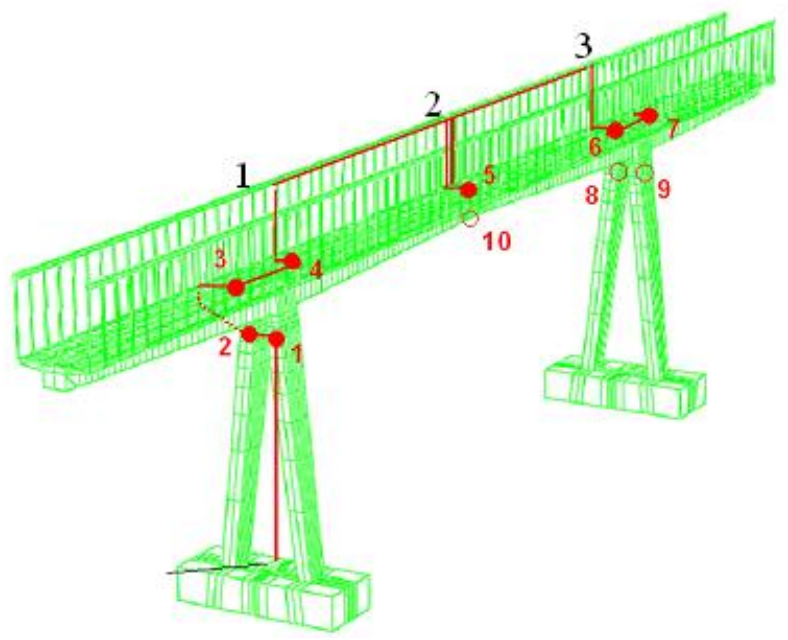

Fig. 2 Position of FBG patches on the bridge shown in red and joined by a line. Solar radiation sensors locations on the top of the deck are shown as black numbers on top of the railings (1,2 and 3).

Fig.s 3 and 4 show typical examples of temperature and strain data before temperature compensation over a one day period. (Sensor 4 is not included here as it was broken soon after installation.) It is clear that all sensors exposed to the direct sun have additional features described here as a fine structure, whereas the ones in the shade do not.

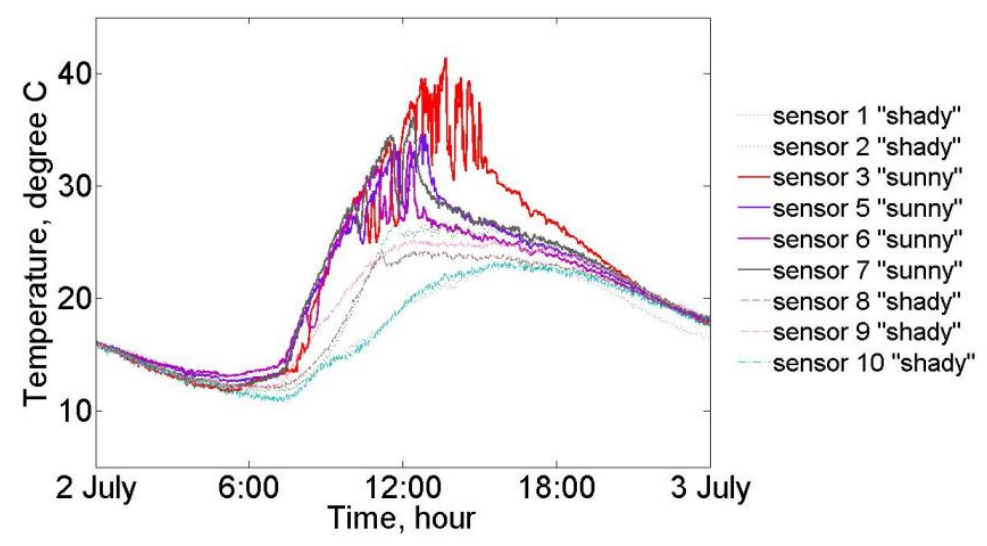

Fig. 3 Temperature for all FBG sensors.

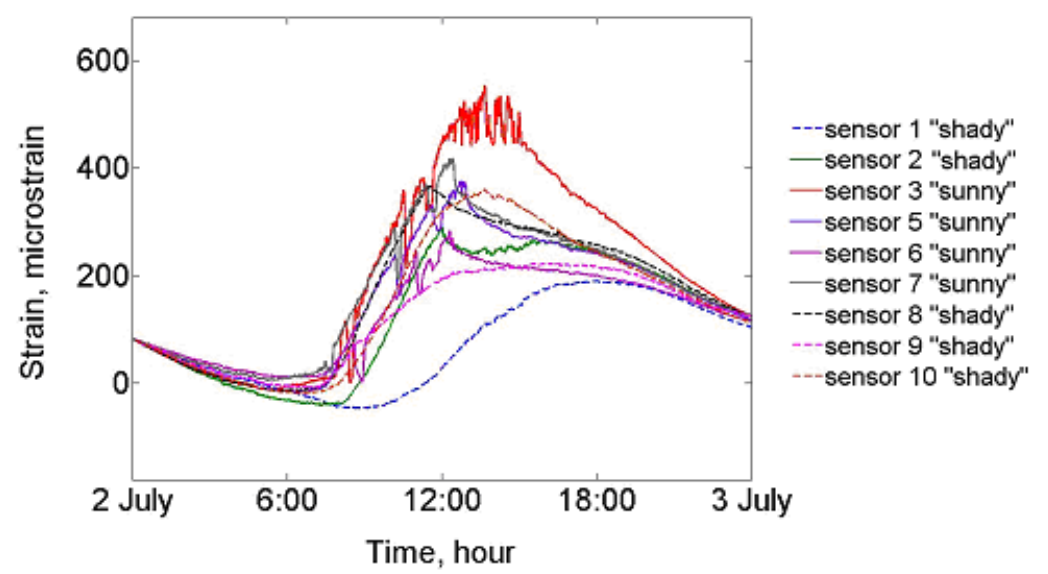

Fig. 4 Strain without temperature compensation for all FBG sensors 
Three solar sensors were positioned along the deck at both ends and in the middle as shown in Fig.2. The FBG temperature data was compared with the solar radiation sensor output. The additional features in the FBG temperature response correspond well with solar radiation measured in similar locations. An example of solar radiation sensor 3 and FBG 7 data is shown in Fig.5.

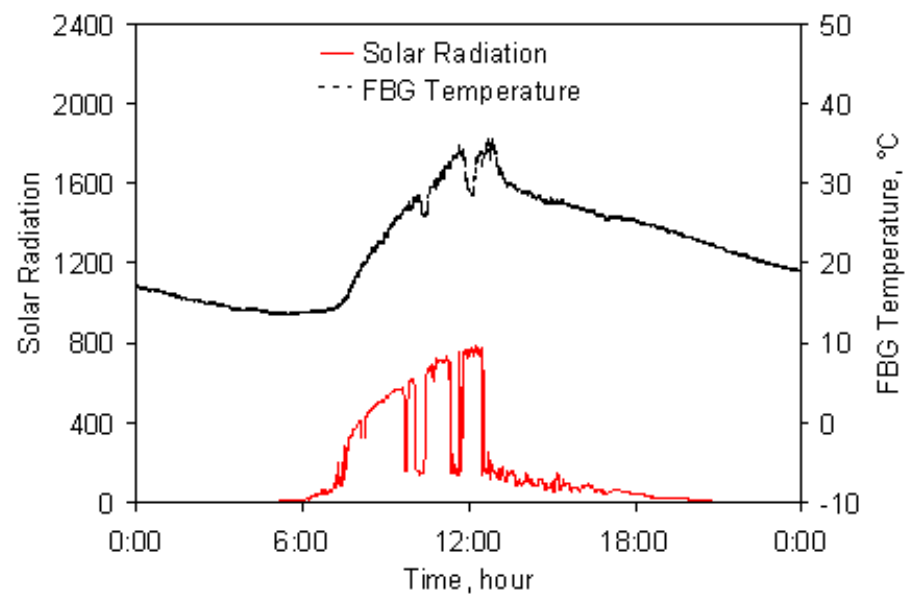

Fig. 5. Typical examples of FBG temperature (line on the top) and solar radiation sensors (line on the bottom).

Moreover, the FBG sensor 3 is located on the sunniest end of the bridge and the time period of solar activity is therefore the longest, as is the time duration of additional fast varying features of the FBG response which lasts until 15.00 in the afternoon, as shown in Fig. 4. (Compare FBG sensor 3 with FBG 5 in the middle of the deck and FBG sensor 7, on the other end of the deck, in Figure 2.) Our conclusion is that the changes in the data are attributable to changes in the solar radiation and not due to noise or poor sensor / installation quality.

\section{Temperature Compensation for a FBG Patch.}

Each patch consists of two Fibre Bragg Gratings. One is used to sense strain and temperature (FBGa) simultaneously and the other only senses temperature (FBGb) because it is not attached but only enclosed in the patch. The two FBGs are close enough to allow their temperatures to be considered the same. The technique for dual FBGs temperature compensation is as follows: the wavelength shift of the FBG1a which senses both temperature and strain, can be given as

$$
\Delta \lambda_{F B G a}=k_{\varepsilon} \varepsilon+k_{1 a} \Delta T
$$

FBG1b is sensitive only to temperature and its wavelength shift is

$$
\Delta \lambda_{F B G b}=k_{T b} \Delta T
$$

where $\Delta \lambda$ is the wavelength shift for FBGa and FBGb respectively, $k_{\varepsilon}$ is strain coefficient and $k_{T a}$ and $k_{T b}$ are coefficients of thermal expansion which were determined experimentally in the laboratory or taken from a datasheet provided by the sensor manufacturer. In this work we calibrated in the laboratory. A typical patch was attached to the concrete block from the bridge step using the same procedure as during installation on the main bridge. Temperature coefficients were obtained experimentally by heating and cooling the specimen in the oven. Temperature compensated strain was calculated by substituting $\Delta T$ from Eq.2 into Eq.1 [3]. It is important to note that for Fibre Bragg gratings, the wavelength shift induced by $1{ }^{\circ} \mathrm{C}$ is almost equivalent to that induced by $10-20 \mu \varepsilon[3]$. 
Time Lag in Temperature Compensation Scheme and Uncertainty.

Traditionally temperature compensation is done at the same moment in time. The sensors in the shade can be reliably temperature compensated using traditional methods. However, the sensors in sunny areas show not only additional features but also a time lag between the strain and temperature responses during morning hours as clearly indicated in Fig. 6 at about 11.30. The time lag appeared to be 13 minutes for the morning data but disappeared in the afternoon data from 13.00 to 15.00 .

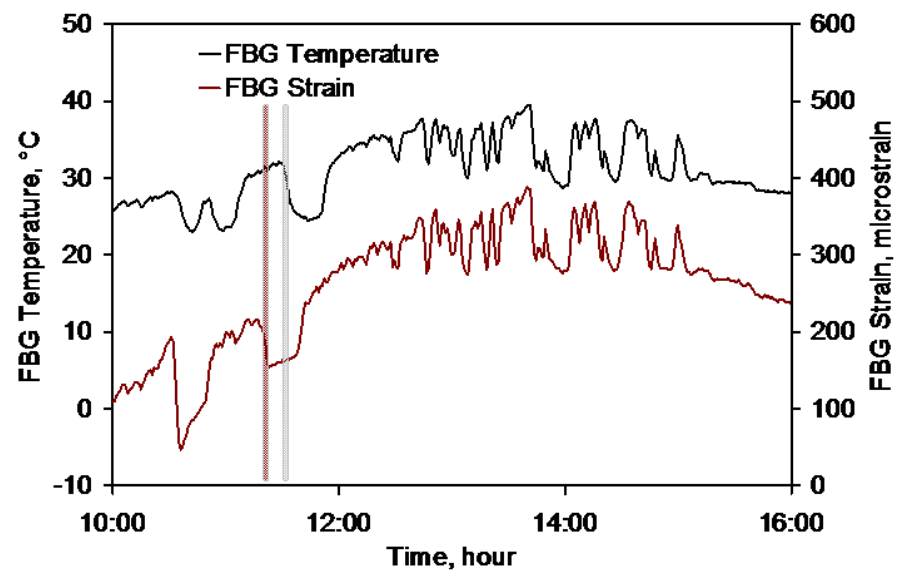

Fig. 6. FBG3 strain and temperature over 6 hours without time lag correction

If the traditional method for temperature compensation is used, strain variation of up to $300 \mu \varepsilon$ (peak to peak) is introduced - shown in Fig 7 by the solid line. The dotted line shows the same data but with temperature compensation including 13 minutes time lag shift correction.

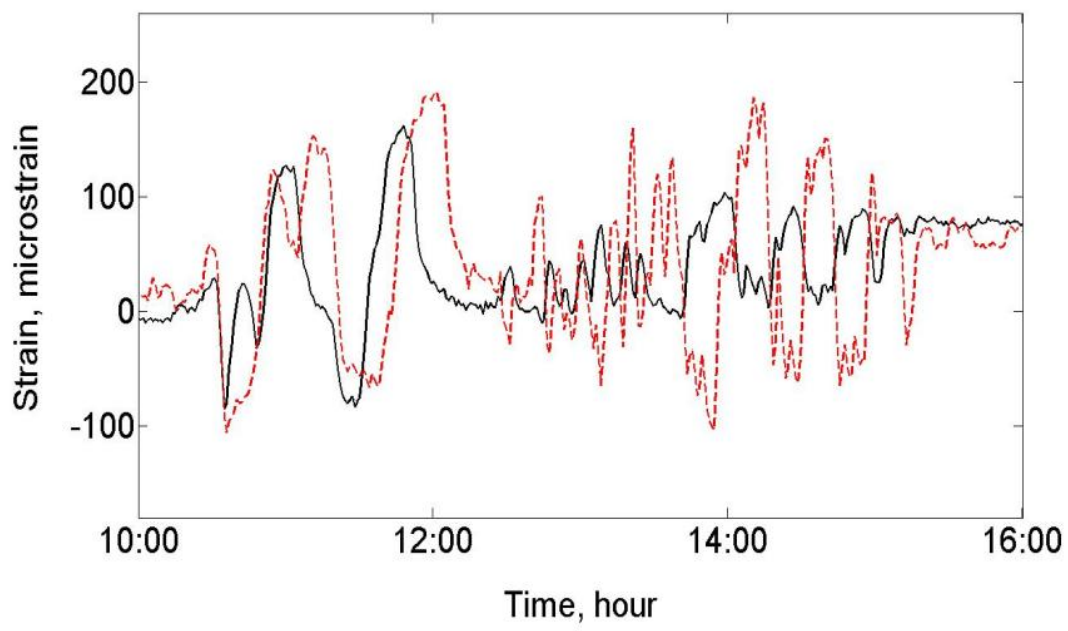

Fig. 7 FBG3 strain data after temperature compensation without time lag correction (solid line) and with 13 minutes time lag correction (dotted line). 


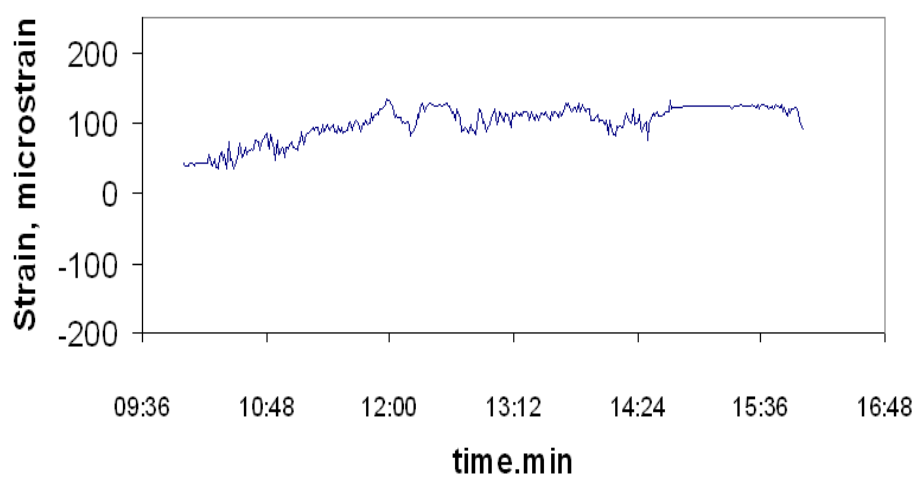

The data from other strain sensors in similar locations (see Fig 8) do not support the strain variations of that magnitude. Therefore, they are likely to be artificial and application of this simple temperature compensation (with or without time lag) could lead to misinterpretation of the data.

Fig. 8 Strain measured using a Vishay electrical resistance strain gauge located next to the sensor FBG 3 on the top of the deck.

Uncertainty in strain measurement using the temperature compensation scheme described above was determined by taking into account the uncertainty in wavelength measurements, temperature and temperature coefficients for both FBG in the patch. Preliminary calculations showed that the uncertainty associated with temperature compensation is the largest contribution to the uncertainty budget.

\section{Summary}

The observed phenomenon in strain measurements using FBG patches relate to changes in temperature due to solar radiation. This, to our knowledge, is a novel interpretation and worth bringing to attention of SHM community. This paper used FBG sensors as examples, but other sensors also show differences between sunny and shady locations. More work is required to understand fully this phenomenon.

Time lag between temperature and strain responses of two FBGs in the same patch presented an interesting challenge. It seems to depend on the rate of change in temperature and could be related to the properties of composite material used for making patches. It needs more experimental investigation in a controlled laboratory environment. At present, our recommendation would be to avoid locations exposed to direct solar radiation.

This paper is part of the continuous work on long-term performance of various sensors in outdoor environment to improve reliability of monitoring systems and interpretation of data.

\section{References}

[1] A,Karimi. Analysis of footbridge under loading, to be published

[2] Sung-Pil Chang etc . Necessity of the bridge health monitoring system to mitigate natural and man-made disasters : Structura and infrastructure Engineering, June 2009, p.173-197.

[3] Information on http://www.npl.shm

[4] Zhi Zhou, OU Jinping. Techniques of temperature compensation for FBG strain sensors: APCOM (2004), p.465-471 